\title{
Changes in dietary consistency and the epidemiological occlusal transition
}

\author{
by Elsa M. Van Ankum
}

Malocclusion is the misalignment of the human dentition and craniofacial complex. Orthodontic treatment to correct this is quite common in modern Western contexts, and has variable prevalence in other areas as well. The archaeological record stands in stark contrast, with most past humans having teeth that align well. What could cause different populations to exhibit these characteristics? There is evidence in both modern and archaeological contexts that levels of dietary masticatory stimulation during development greatly change occlusal characteristics. Additional bite force and number of chewing cycles creates a flatter Curve of Spee (COS), greater subnasal prognathism, and increased progressive tooth wear, which together facilitate better occlusion. With dietary changes such as those seen in the Industrial Revolution, the comparatively softer food creates widespread malocclusion in the affected population. In this article I explore these ideas in relation to archaeological and contemporary case studies. Health transitions such as this commonly coincide with major changes to subsistence style, and can help biological anthropologists better understand the experiences of those in the past.

\section{Introduction}

Today, orthodontic correctional treatment is an expensive albeit important part of many people's lives. The main goal is to correct malocclusions in the dentition, or in other words, to align the teeth and jaws. Because of the ubiquity of this correction in the modern Western world, one might wonder why we do not see the need for it in the vast majority of the ancient world. While in the past there may be the occasional individual with a misaligned orofacial complex, today it is relatively rare for a post-industrial adolescent not to need some form of dental intervention. There is strong evidence that the reason for this is diet. Increased bite force and number of chewing cycles during development, stimulated by a tough/abrasive diet, creates a flatter Curve of Spee (COS), greater subnasal prognathism, and

Elsa M. Van Ankum is a BA (Honour) student at the University of Alberta (Department of Anthropology (1315 HM Tory Building, Edmonton, Alberta, T6G 2H4 [vanankum@ualberta.ca]). increased tooth wear (Ingervall and Bitsanis 1987; Kaifu et al. 2003; Laird et al. 2016; Lieberman et al. 2004). This suite of traits facilitates better dental and skeletal occlusion; this is why the modern post-industrial diet results in an epidemiological transition of occlusion and subsequent significantly increased malocclusion in modern populations. An explanation of the dietary transition will follow a treatment of the factors that affect malocclusion. These theories will then be explored in relation to several archaeological case studies, and an outline of how malocclusion and diet should be studied on skeletal populations. Consideration will also be given to future research in this growing field.

\section{Factors in the Etiology of Malocclusion}

Based on the length and scope of this paper, only Malocclusion can be defined as an incorrect relationship between the dental arches, the teeth, or both (Corruccini 1984). It is typically referred to as 'skeletal malocclusion' when the main issue 
is the relationship between the dental arches, and 'dental malocclusion' when problems are caused by tooth crowding (Corruccini 1984). For the purposes of this paper, the clinical definitions and classes of malocclusion will not be addressed, and skeletal and dental malocclusions will be combined and considered together. This allows archaeological and clinical literature to be compared. Similarly, there are a number of occlusal curves, not all of which will be discussed here. The COS is the occlusal curve when viewed in the sagittal plane of the permanent dentition, measured as the arc between the buccal mandibular first molar cusp and either the incisal edges or the tip of the canine, depending on the source (Marshall et al. 2008) (fig. 1). There is also a measurement called the Curve of Wilson, which exists on the bucco-lingual axis of the molars; these arcs combine to create the helicoidal Sphere of Monson (Tobias 1980). This sphere is used to describe the dynamic motions in the oral cavity during mastication, and is affected by occlusion. While the helicoidal sphere and Curve of Wilson are important in mastication, only COS will be considered here, as the scope of this paper is suited best to address the COS and its relationship to orofacial morphology. With those considerations, here is a treatment of these features.

The COS is greatly influenced by prognathism (Laird et al. 2016; Tobias 1980). Both prognathism and COS are interrelated with mandibular morphology (Tobias 1980). Prognathism describes a face with a protruding subnasal region, and is often considered when discussing differences between modern humans and our extinct hominid ancestors. A highly prognathic individual has a projecting mandible

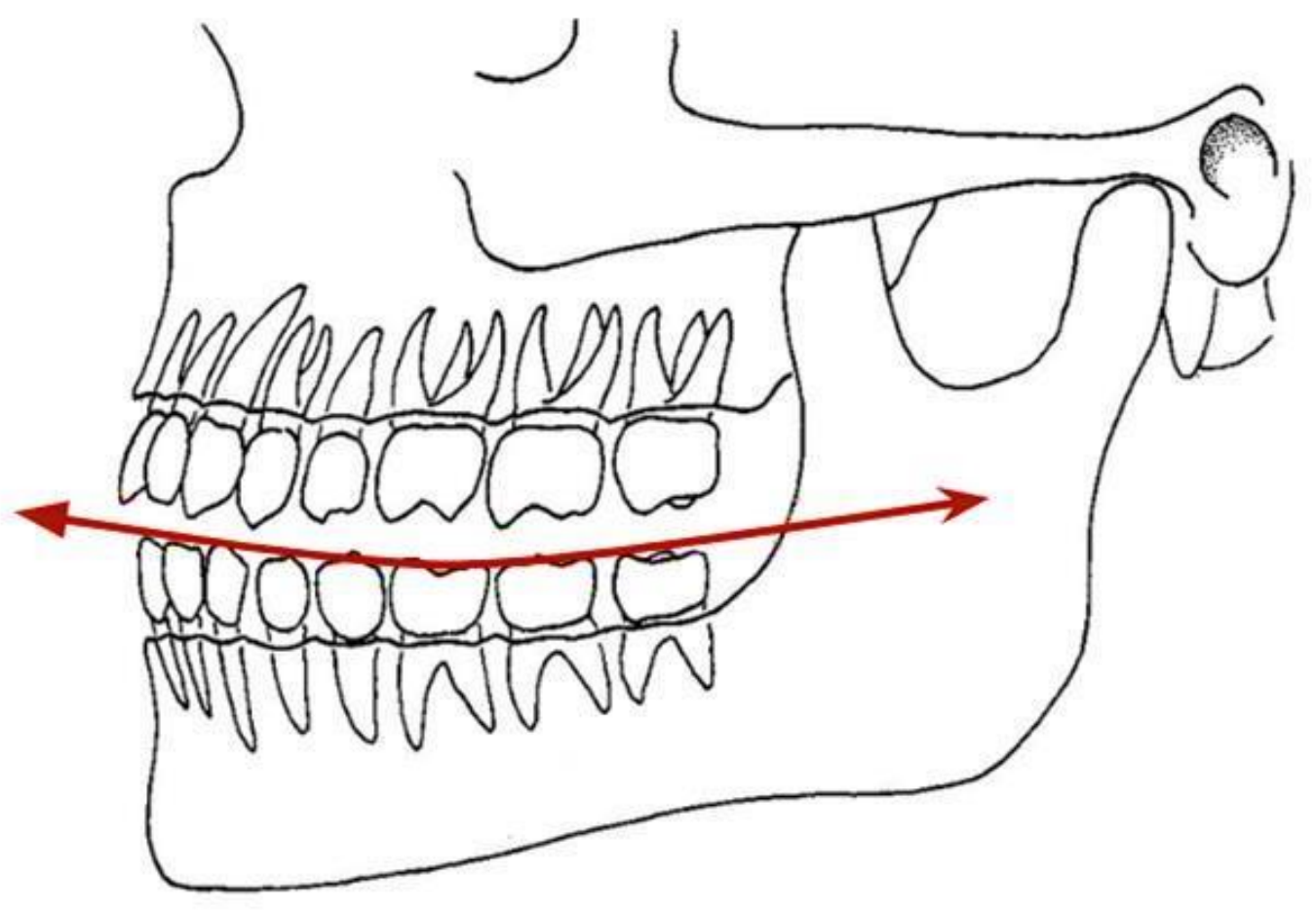

Figure 1. A lateral view of the mandible and maxilla, with the red line representing the Curve of Spee (COS). Note that the line touches the mandibular buccal molar cusps and the tip of the canine. Original image: MouseDoctor 1970. 


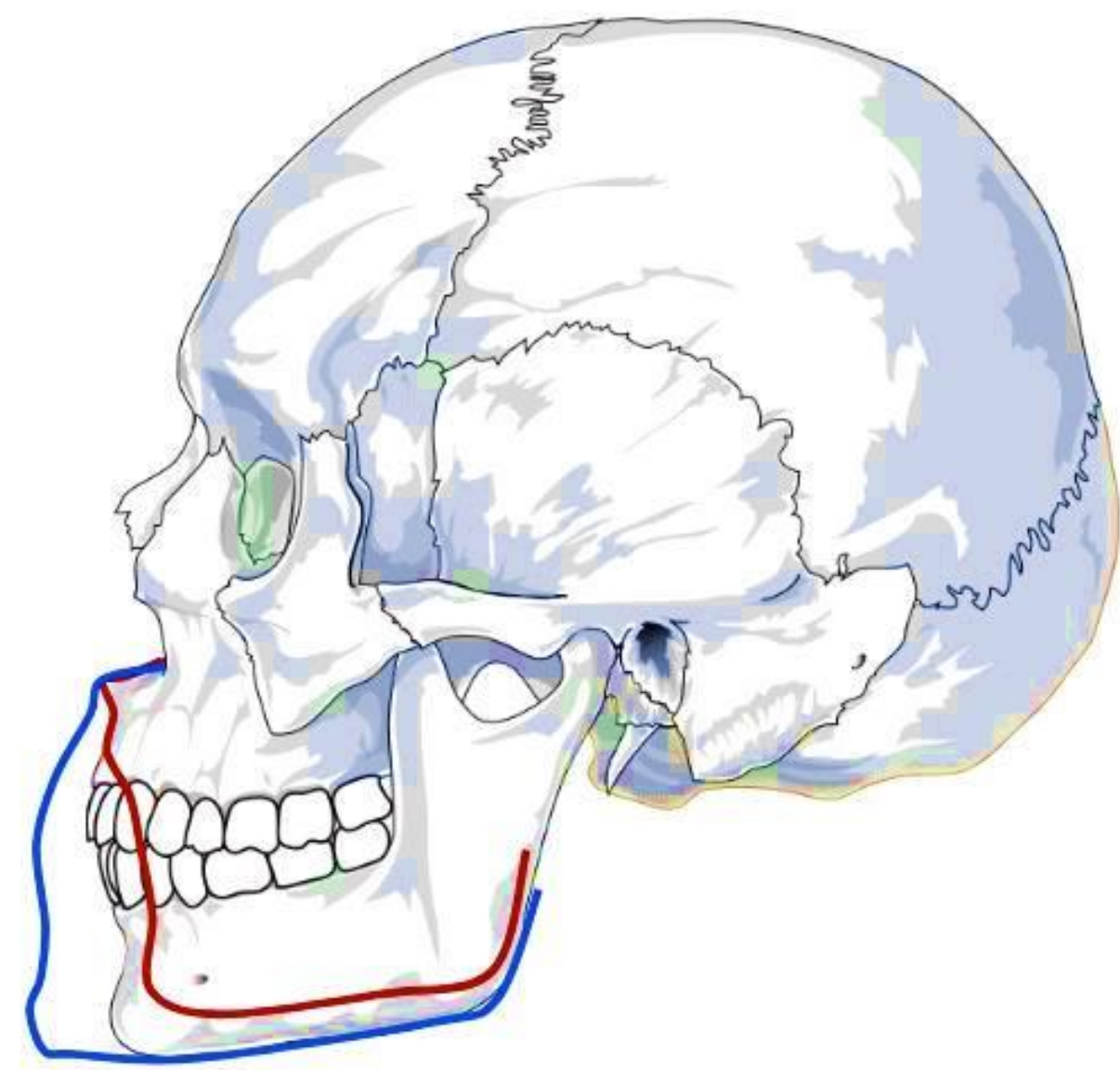

Figure 2. Lateral view of the skull. The red line represents an example of orthognathism, with the lower face being shifted posteriorly under the orbits. The blue line is a representation of prognathism, with the lower face protruding anteriorly. Image from: https://www.wpclipart.com/signs_symbol/skull/Human_skull_side_view.png.html.

and maxilla, while an orthognathic individual's lower face is situated farther posteriorly, bringing it into vertical alignment with the orbits (fig. 2). Laird and colleagues (2016) conducted a geometric-morphometric study correlating COS and prognathism in modern humans and archaic Homo sapiens. Their results showed that while modern humans were as a whole more orthognathic, there are still differences between populations. COS and alveolar prognathism covaried, so people with deeply curved COS are orthognathic, while those with flattened COS were more prognathic (Laird et al. 2016:233).
While their analysis is robust, their conclusion that COS and prognathism is a populationspecific marker in modern humans may need to be revisited (Laird et al. 2016:233). The modern groups tested were European-American and African-American, and their claim that this was indicative of those groups seems to support a genetic and potentially problematic idea of craniometric variability. Populations are defined based on social factors as well, so it would be prudent to examine the diets that the individuals were raised on. For reasons that will be discussed more in depth in the section 'The 


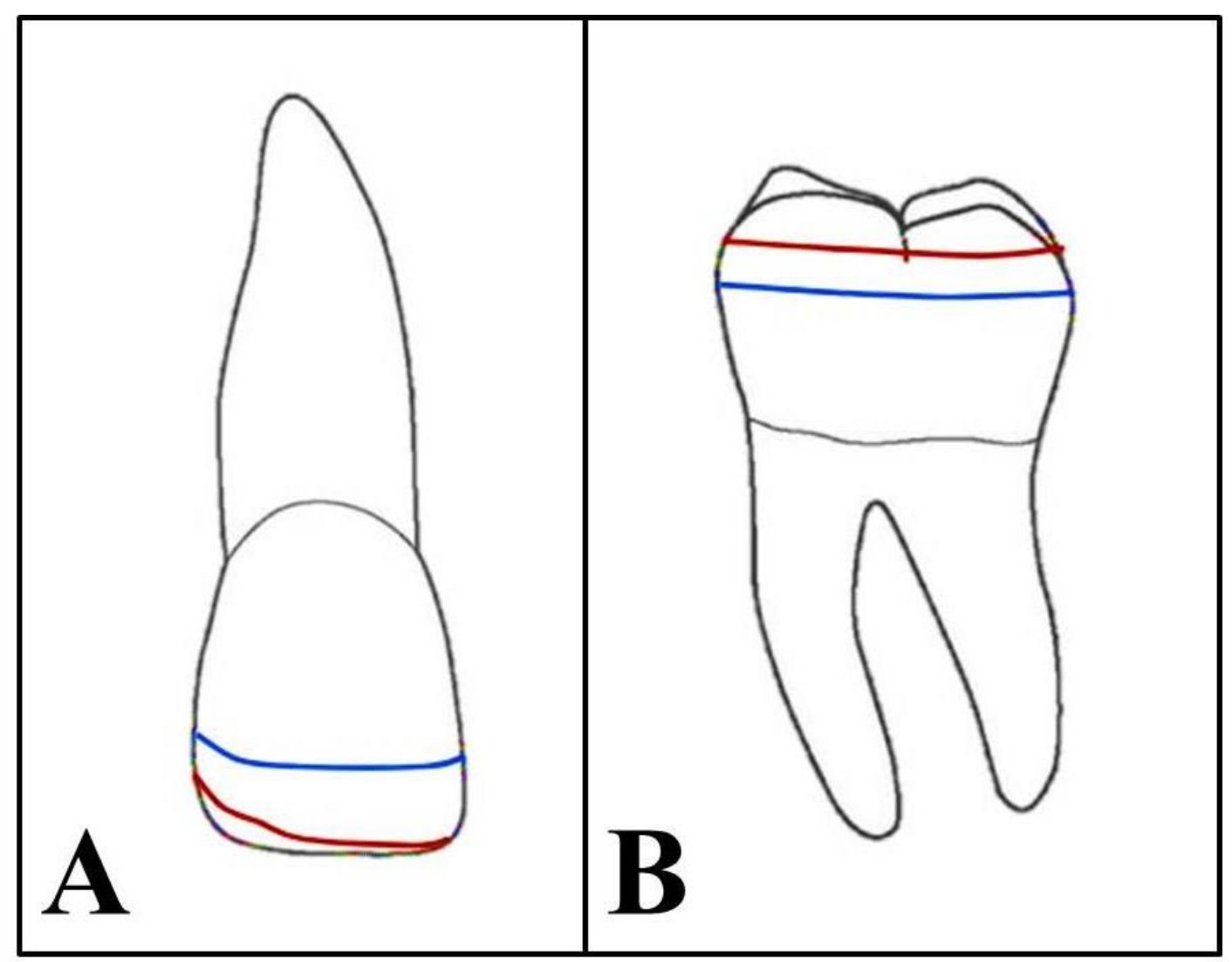

Figure 3. A maxillary first incisor (A) and a mandibular second molar (B). Lines represent occlusal crown macrowear progression throughout time in a hypothetical person with normal occlusion. The red lines show this theoretical person's crown at 20 years after eruption, and the blue lines show the crown at 40 years after eruption. This graphic is intended to show the idiosyncratic nature of macrowear, as well as visualize its possible progression. Original images from: Whatwhenhow 2018 (Part 2 and Part 3).

Epidemiological Occlusal Transition,' environment plays a vital role in the etiology of mandibular morphology. The Hamann-Todd collection, a modern assemblage collected in the early 1900s, was used for this analysis. These remains do not include any information on what socio-economic environment people grew up in, and therefore what kind of diet they had access to (Cleveland Museum of Natural History 2018). As this may influence the orofacial complex that is being studied, there is therefore reason to review any conclusions based off this collection. Even with this caveat, the work of Laird et al. (2016) accurately exemplifies the relationship between COS and subnasal prognathism.

The final factor to be discussed here is tooth wear. Abrasion is the macrowear that occurs due to mechanical friction from food or other items in the mouth. Attrition is macrowear generated from tooth-on-tooth wear, either between adjacent teeth (interproximal) or occluding teeth. Figure 3 shows the cumulative effects of abrasion and attrition on the occlusal plane. Microwear and interproximal wear will not be considered in this paper, as there is no evidence that either is extensively correlated to malocclusion and diet 
(Sengupta et al. 1999). It is generally assumed that the majority of occlusal macrowear is generated when masticating food, with the exception being those individuals who grind their teeth for psychological reasons (Kaifu et al. 2003). Sengupta and colleagues (1999) conducted a study looking at occlusal macrowear, third molar presence, and COS. The modern dentition is associated with a greater frequency of third molar impaction and agenesis, as well as modest wear rates when compared with typical preIndustrialized dentitions (Boughner 2017; Kaifu et al. 2003; von Cramon-Taubadel 2011). Owing to this, the authors hypothesize that increased rates of wear in the past, due to abrasive diets, maintained a flattened COS. Their rationale that any modern wear is caused by prolonged use-life rather than abrasive diets is most likely a robust assumption (Kaifu et al. 2003). However, results indicated that there was no straightforward relationship between COS and macrowear; COS was not maintained by wear. While the authors' goal was to determine if a deeply arched COS was necessary to include in the design of dental prostheses, this evidence can also be interpreted differently. Given that mandibular structure is generally determined by the time that the dentition shows heavy wear, it is unlikely that COS depth can be greatly affected by that wear. Given this, I believe it is reasonable to conclude that wear and COS both result from one general cause: mastication of food. By chewing abrasive and tough foods during growth, mandibular morphology is altered to create a flatter COS; if a similar diet is consumed in adulthood, more severe wear may be seen in adulthood. Tooth wear and COS depth are likely related, but perhaps not in the manner hypothesized by Sengupta and associates (1999). This idea will be explored further in the following section.
As defined above, it is clear that mandibular COS, subnasal prognathism, and occlusal macrowear are interrelated with each other and malocclusion (Baydaş et al. 2004; Kaifu et al. 2003; Sengupta et al. 1999; von CramonTaubadel 2011). The degree of prognathism determines how deeply arced the COS is, and both COS and wear are potentially influenced by similar biomechanical stress (Laird et al. 2016; Sengupta et al. 1999). The morphological changes - including gonial angle, arch shape, and depth - occurring in the mandible and maxilla that determine prognathism and COS are a large part of what facilitates normal occlusion. Comparatively spacious alveolar surfaces in prognathic individuals support the development of an uncrowded permanent dentition, and this in turn facilitates a proper relationship between the dental arches. Attrition and abrasion cause tooth wear, which is exacerbated by food consistency and mastication habits. Therefore, the question becomes: why is malocclusion so prevalent in modern Western society?

\section{The Epidemiological Occlusal Transition}

The answer to this question lies in dietary change. There have been several subsistence revolutions that we know of in human existence. Changes associated with these transitions had far reaching implications, beyond merely what foods were included in the diet. Social structure, sedentism, animal husbandry, domestication, trade relations, settlement patterns, and material culture are all affected by alterations in food production and cultivation (Laird et al. 2016; Pinhasi, Eshed, and von Cramon-Taladel 2015; Rando, Hillson, and Antoine 2014). Paleopathology is one way that these changes can be observed osteologically. 
Osteological analysis allows researchers to see evidence of changes in health that, when analysed demographically, can inform us about societal change. The Neolithic Agricultural transition is viewed as the first of these important revolutions, which occurred thousands of years ago at various locales and specific times in different cultures, if it occurred at all (Pinhasi, Eshed, and von Cramon-Taladel 2015). Subsequent to this, the Industrial Revolution occurred in the Western world in the $18^{\text {th }}$ to $19^{\text {th }}$ centuries. This second shift was characterised by drastic increases in urbanization and technological advances in production, and still has a radical effect on health and disease today (Corruccini 1984; Komlos and A'Hearn 2017; Rando, Hillson, and Antoine 2014). While both subsistence revolutions likely resulted in dental changes, I am interested in the micro-evolution that is associated with the postindustrial diet and modern clinical malocclusion, and will therefore focus only on the Industrial Revolution.

As a model for pre-Industrial diet, what follows will be a highly generalized treatment regarding the texture and consistency associated with agricultural and hunter-gatherer subsistence methods. This paper is by no means an exhaustive contemplation of the intricacies involved with regional and cultural variation seen in diets, and it is acknowledged that each population has the potential to experience these changes associated with the Industrial Revolution in a myriad of ways. This generalization is used to emphasize that the consistency of food has generally been altered by said transition, and that this has resulted in a proportional change to dentition and orofacial complex. This model can be used to assess individual assemblages for similar changes, and in those cases, it would be necessary to carry out dietary analysis in the form of artifact analysis, stable isotope analysis, paleobotanical analysis, and faunal assemblage analysis in order to quantify dietary change and the implications for that society. For the purposes of this paper, this generalization is a necessary step so that this broad epidemiological transition can be conceptualized.

As a model for prototypical pre-industrial fare, medieval English diet will be reviewed here. Diets were coarse and tough, often consisting mainly of bread and beer (Rando, Hillson, and Antoine 2014:77). Most people were agriculturalists who supplemented their diets with gathered wild food when needed, wherein upper classes consumed more cultivated foods than lower classes. Along with bread, pottage - a thick, stew-like dish which included grains and vegetables - was eaten by everyone. Processed, sugar-rich food was essentially unavailable during this time. Abrasive inclusions in ground flour and tough unprocessed food contributed significantly to a coarse, abrasive dietary texture. In contrast, post-Industrial Revolution populations consumed a very different set of foods. During the 17th century, changes to food consumption, availability, and production resulted in a considerable increase in sugar consumption and the use of finely milled flour (Rando, Hillson, and Antoine 2014:78). These dietary alterations continued throughout the $18^{\text {th }}$ and $19^{\text {th }}$ centuries, with staple foods becoming increasingly mass-produced outside the home and more easily purchased. In short, the tough and abrasive components from unprocessed, homemade food have been largely replaced by softer, less textured, and easy to masticate products.

These changes in dietary consistency alter orofacial morphology, and therefore also occlusion. This is because of its impact on mastication, and subsequently on the biomechanical strain inflicted on the jaw. There is a difference between tough and abrasive foods, which impact the strain: tough food (e.g. meat) 
requires more bite force and many chewing cycles before swallowing, whereas abrasive foods (e.g. nuts) do not necessarily require more force or chewing, but rather creates higher levels of friction and wear per bite (Ingervall and Bitsanis 1987). Bite force is important because it is a measure of how hard the muscles strain in order to chew food (Ingervall and Bitsanis 1987; Ledogar et al. 2016). The stress created by bite force affects the development and maintenance of alveolar bone. There is evidence that the COS has a role in determining bite force potential, as a deeper curve can increase the force potential for each chewing cycle (Fueki, Yoshida, and Igarashi 2013b; Ingervall and Bitsanis 1987). A chewing cycle is the movement of the bolus of food around the mouth, measured by a single occlusion of the opposing dental arcades (Fueki, Yoshida, and Igarashi 2013a). Certain foods require more cycles to process, which has implications for muscle use and stimulation of orofacial bones. There is evidence that jaw morphology greatly influences an individual's biological potential to perform each of these aspects. Greater subnasal prognathism, facilitated by a more robust jaw and a more acute gonial angle, has been implicated in the ability to perform more chewing cycles at a higher bite force (Ingervall and Bitsanis 1987; Fueki, Yoshida, and Igarashi 2013a, 2013b). This suite of traits would facilitate better occlusion, and lead to more severe levels of tooth macrowear. These bone and muscle factors are important for developing and maintaining a normal dental complex, and are greatly influenced by diet.

The ability to efficiently process tough food is greatly influenced by the diet consumed during development (Boughner 2017; Lieberman et al. 2004; Limme 2010). Permanent dentition is almost fully erupted by late adolescence, with the variable exception of third molars. The jaw has developed into what will most likely be its adult form, with no significant morphological or metric changes likely to occur until senescence (Scheuer and Black 2004). The idea of functional integration can be used to explain this. Boughner (2017) examined the biomechanical relevance of this concept in relation to the epidemiological occlusal transition. She investigated the effects of mastication on orofacial development, concluding that modern children who consume more processed diets go on to experience higher rates of malocclusion, temporomandibular joint disorders, and tooth impaction (Boughner 2017). The stimulation from bite force that begins soon after weaning plays a large role in the eventual morphology and functional capacity of the mouth as a whole, which is why diet at this young age is so pivotal. Functional integration is the idea that human jaws are evolved to masticate to a certain level, and that by not using them for this function from a young age, we are causing an interruption in tissue development. While the effects of biomechanical forces on growing musculoskeletal tissues of the face are not well understood, it is suggested that introducing hard foods - such as raw vegetables, nuts, and fruits - could minimize malocclusion and joint disorders (Boughner 2017). This has important implications for determining health policies and parenting advice, because altering dietary consistency could possibly lessen the need for orthodontic treatment. Boughner (2017) does note that although malocclusion is treated as pathology in modern Western society, it is not always inherently detrimental to mortality or morbidity. Though severe cases can make chewing difficult, changes to the diet or method of food preparation usually means that even highly affected individuals are able to consume the required nutrients. Additionally, malocclusion can cause poor oral health by facilitating plaque build-up 
and development of dental caries, though modern hygiene practices usually mitigate the permanent damage caused by this (Feldens et al. 2015). The seemingly minimal health consequences may seem counter-intuitive in today's society, as orthodontic adjustments are so common. The reason for their pervasiveness in modern society is often sociocultural rather than biological. As is commonly observed, the aesthetic component of a normally occluded dentition is important to many humans, because smiles are a large part of how people present themselves. The smile can influence social signals and self-confidence, which explains why malocclusion is often a concern to modern Western society (Naini et al. 2006; Rosenstiel and Rashid 2002; Sarver 2001; Witt and Flores-Mir 2011). Therefore, it is evident that diet during development can clearly influence occlusion and orofacial development, impacting future quality of life both biologically and socially.

Given that post-Industrial diets did not stimulate developing jaws to facilitate effective growth, these changes can be seen especially in mandibular form and corresponding occlusal variables. The mandible is more plastic than the maxilla, meaning that it is more likely to exhibit phenotypic changes influenced by the environment (Pinhasi, Eshed, and von CramonTaladel 2015). It is expected that there are more diagnostic changes seen in the mandible, and therefore maxillary changes - though often present - will not be reviewed here. Fueki and colleagues (2013a, 2013b) used a set of experimental studies on permanent dentitions on living humans to measure the diagnostic changes in the mandible. There is significant correlation with a flatter COS and the ability to effectively mix and comminute food (Fueki, Yoshida, and Igarashi 2013a). This flatter COS results in a larger occlusal sphere diameter, which is especially conducive to mastication efficiency.
Fueki and colleagues (2013b) also confirm that occlusal sphere diameter, as facilitated by depth of the COS, can be used to predict biomechanical parameters involved with mastication. For all test foods, which ranged in consistency, a flatter COS produced faster closing velocity. It is not unreasonable to deduce from these studies that dietary consistency during development would highly influence and adult's ability to masticate food. The relationship between mandibular morphology and biomechanical stress dictates that the mandibular growth occurs on the plane of deformation (Lieberman et al. 2004). Lieberman and colleagues (2004) conducted experiments to measure mandibular growth according to dietary consistency in the rock hyrax, an animal that has similar dynamic jaw movement and force distribution as humans. They found that the mandible grew according to the strain exacted on it by food consistency. Lieberman et al. (2004) conclude that adult dentition has the form that is best suited for mastication according to the diet experienced during formation, similar to Fueki and colleagues (2013a, 2013b). This is highly supportive of the idea that deep COS and corresponding orthognathism in adults is indicative of a softer diet during development (Laird et al. 2016; Lieberman et al. 2004).

Furthermore, the mandibular morphological relationship to diet is reflected in the gonial angle. In living children, Ingervall and Bitsanis (1987) observed that weak bite force was correlated strongly with 'long-face' morphology. This morphology is defined by a highly obtuse gonial angle and posterior rotation of the mandible. As their experimentation showed, this morphology is symptomatic of under-stimulation of the masticatory musculoskeletal system. In their study the children were 7 to 12 years old, meaning that mature mandibular morphology was still under establishment (Ingervall and Bitsanis 1987). Therefore, vigorous mastication of sticky 
pine resin for over a year caused a reversal of the bite force and associated morphology in nine out of twelve children (Ingervall and Bitsanis 1987). As a result of the dietary change, children's mandibles rotated anteriorly, facilitated by increased gonial angle and stronger chewing muscles (Ingervall and Bitsanis 1987). These changes have also been observed in more recent studies of mandibular metrics and masticatory muscles (Sella-Tunis et al. 2018). Based on the studies outlined above, dietary consistency has a clear impact on masticatory stimulation, specifically in relation to gonial angle and facial form.

Mandibular morphological characteristics, including COS, prognathism, and gonial angle, are determining factors in the etiology of malocclusion (Boughner 2017). Tooth macrowear has often also been included in the suite of traits that facilitate proper occlusion (Kaifu et al. 2003). However, as explored previously, mandibular morphology is more clearly correlated with changes in occlusion. As tooth wear does not form until long after malocclusion has developed, it can instead be taken as a symptom indicative of the diet which formed the malocclusion (Kaifu et al. 2003). In this way, the correlation of severe tooth wear and normal occlusion is actually indicative of a tough diet that facilitated both the wear and the occlusion. Similarly, lack of tooth wear and poor occlusion are both are reflective of the diet, but the actual wear does not facilitate malocclusion in the way that diet does. Changes in a population's dietary consistency affect development of the orofacial complex, which in turn leads to higher frequencies of malocclusion within that population; this transition can be termed 'the epidemiological occlusal transition' (Corruccini 1984).

\section{The Epidemiological Occlusal Transition}

Some researchers support that environmental components, such as diet, are not the main etiological factor behind malocclusion (Eguchi et al. 2004; Normando et al. 2011). Their arguments focus on genetics and the heredity of dental misalignments. Normando et al. (2011) investigated incidences of malocclusion in two indigenous Amazonian villages. One village was derived from a single couple having split off from the original village generations ago. The founder population had much more frequent malocclusions than the original, which the authors used to conclude that genetics had a greater impact on malocclusion than environment did (Normando et al. 2011). However, their findings indicated that each population had different types of malocclusion, which leads one to believe that genetic and environmental influences act on different types of malocclusions in different ways.

The concept of malocclusion heredity has also been tested in monozygotic twin studies (Eguchi et al. 2004; Townsend et al. 2009). The benefit of analyzing monozygotic twins for specific factors is that twins have nearly identical genetics, and so any variation that is seen will be the result of the environment. Researchers are then able to estimate the genetic and environmental components that contribute to a certain phenotype or factor. Townsend and colleagues (2009:46) reviewed twin studies for incidences of malocclusion, and suggested a fundamental critique of the approach: if twins were raised in the same environment, how can researchers separate external influence from genetic ones? If the diet consumed during development is as important for malocclusion as is postulated, then twin studies, where both are 
raised in the same environment, are insufficient for examining the genetic contribution to the issue. Additionally, it is clear that phenotype is not merely the result of nature or nurture, but nature, nurture, and the interaction of both (Harris 2008; Townsend et al. 2009). Knowing these criticisms of the genetic experimental model, this paper will now discuss the results.

In their review, Townsend et al. (2009) determine that some occlusal variables are more heritable than others. For instance, tooth size is largely inherited, but most other occlusal variables are heavily influenced by the environment. These findings agree with those put forward by King and colleagues (1993). The authors used a longitudinal study of siblings to investigate why siblings commonly present with similar malocclusions. This observation has long been used to support a genetic cause of malocclusion; however, King and colleagues (1993) suggest that these siblings are raised in similar environments, and so it is likely that they will respond to their environment in similar ways. An important thing to consider is that there are different types of malocclusion. Misalignments are formed by skeletal variables interacting with dental variables, which occur to differing degrees under variable conditions. The results of King and colleagues (1993) support that genes and the environment can affect some variables more than others and that the skeletal variables are more heritable than dental variables. King and colleagues (1993) determined that malocclusions are largely environmentally influenced, although genetic potential does impact it as well. Townsend and colleagues (2009), whose review of twin studies contains similar findings, express support for this idea as well, saying that "the extent to which genes determine a trait has no relationship whatsoever with the success of environmental intervention" (Smith and Bailit 1977:73, quoted in Townsend et al. 2009:S46).
Therefore, it is clear that both genetics and environmental factors contribute to malocclusions. Different types of malocclusion are influenced more by heredity, because occlusal and orofacial variables differ in susceptibility. Genetics, the environment, and epigenetics each contribute to the etiology of malocclusion; however, it is not well understood as to what extent these three factors and their interactions specifically affect the orofacial complex. There is some evidence that some types of orofacial misalignments, such as those seen with the extremely projecting Habsburg jaw, are more related to genetics (Boughner 2017; Harris and Johnson 1991). However these hereditary examples are exceptions and most of the malocclusions seen today are due to environmental influences, such as dietary consistency during development. Genetics would not cause the same rapid and drastic changes we see with dietary change, and dietary findings are corroborated by in vivo studies with animal models (Ciochon, Nisbett, and Corruccini1997; Larsson et al. 2005; Lieberman et al. 2004). We can also see how the diet changes occlusion in archaeological areas with known dietary change, discussed in the following section.

\section{Archaeological Examples}

One way to test how dietary consistency impacts malocclusion, is to use archaeological case studies. Ideally, study populations will originate from the same culture and region, but from different time periods that correlate to 'before' and 'after' a major dietary transition. Often these dietary transitions are not well-documented, and other means are required to establish dietary variation (e.g. stable isotope analysis). By comparatively analyzing malocclusion and mandibular dimensions in large population samples, researchers can see how the theoretical 
and experimental dietary changes discussed above actually affect people.

Varrela (1990) examined the possibility that a malocclusion epidemiological transition was caused by the Industrial Revolution in Finland. A medieval graveyard in Turku consisted of 207 adult individuals, representing lower to middle class Finnish-speaking citizens from the $15^{\text {th }}$ and $16^{\text {th }}$ centuries (Varrela 1990:243). Maxillae and mandibles (141 and 159 respectively, with 93 pairs) were examined for signs of malocclusion, which was considered to be improper bite relationships between available paired jaws, crowding and rotation of teeth, and impaction. These data were then compared with modern Finnish dental information. While the author acknowledged that this archaeological population is not necessarily representative of medieval Finland as a whole, he did emphasize that this was an appropriate situation to examine dietary related changes in occlusion (Varrela 1990).

Although no medieval dietary information was specified, occlusal macrowear was used as a dietary proxy, with high levels of abrasion indicating typical Scandinavian diets from that time period (Varrela 1990). In particular, Varrela (1990) noted that wear on the cheek teeth was more indicative of diet than wear on the incisors, which doubled as a tool and as a result collected extra-masticatory wear accordingly. Therefore, the author stated that the extensive wear on the premolars and molars reflected a rough diet requiring high rates of mastication, indicative of a prototypical Pre-Industrial diet. When compared with modern Finnish dental data archaeological dentition had significantly less variable occlusion (Varella 1990). Overall, the more modern dentitions had higher rates of malocclusions and improper tooth and arch relationships (Varrela 1990). Traits such as overbite, crowding, and crossbite occurred far less in the medieval population than in the post-industrial sample. This is attributed to a shift towards soft and nonabrasive diets in the modern era, as part of the epidemiological occlusal transition model proposed by Corruccini (1984). Though Varrella (1990) does not conduct a very thorough review of evidence for past dietary toughness, this is still a valuable example of the effect that diet can have on occlusion. It would be interesting to conduct a study on the size and morphology of these medieval maxillae and mandibles with comparative data available in a contemporary population. Theoretically, if one were able to obtain consent and ethical clearance, the use of any incidental radiographs of mandibles could be useful to assess gonial angle, robusticity, and rami and body dimensions.

In another study of post-Industrial masticatory changes, Rando and colleagues (2014) examined changes in a London population. Medieval (1050-1550 CE) and postmedieval (1550-1850 CE) adult skeletons were selected from the Museum of London Centre for Bioarchaeology, with 279 individuals chosen from sites in use during one of the aforementioned periods (Rando, Hillson, and Antoine 2014). The authors investigated the effects of modern Western diet on the masticatory complex in terms of orofacial variables. The medieval diet described was typical of preIndustrial agriculturalists in general toughness, abrasiveness, and requirement for extensive mastication (Rando, Hillson, and Antoine 2014). Post-medieval diet required much less dental processing and resulted in far less tooth macrowear (Rando, Hillson, and Antoine 2014). The study included metric analysis of the breadth of the rami, height of the rami, body length, gonial angle, height of the mental eminence, bicondylar breadth, bigonial width, anteriorposterior width, and medio-lateral length of the 
mandibles (Rando, Hillson, and Antoine 2014: 75). This allowed the researchers to view the impact of this dietary change on a London population.

Results from this study indicated that there was a pronounced change in almost all measurements studied (Rando, Hillson, and Antoine 2014). The most substantial differences manifested in the form of a reduction in mandibular width and ramus height, and the gonial angle becoming more obtuse. These changes correspond to individuals with the typical weak bite-force morphology, termed "long-face" form (Rando, Hillson, and Antoine 2014:76). Additionally, the findings corroborate other medieval European studies of masticatory alterations affecting orofacial form. This is especially important because the authors actually considered the sexes separately, and found them to have both changed in the same ways. Their findings support the concept that that sexual dimorphism does not play a part in the etiology of malocclusion, and that diet is the main impetus causing this change. Though the authors did not specifically discuss malocclusion, there are clear links between misalignment and mandibular morphology (Katz, Grote, and Weaver 2017; Rando, Hillson, and Antoine 2014; von CramonTaubadel 2011). Not only can mandibular understimulation cause skeletal malocclusion, but the previously discussed changes in craniofacial metrics and proportions can affect the teeth as well. As discussed above, if an adequately stimulating diet is not consumed during growth and development, the resultant deep COS and low levels of prognathism are more conducive to tooth crowding and rotation in addition to abnormal arch relationships.

Pre- and post- Industrial Norwegian populations have also been analyzed for rates of malocclusion, with differences and findings being surprisingly well addressed in the literature
(Evensen and Øgaard 2007; Lindsten et al. 2002). Both analyses addressed secular trends in dental misalignments between medieval and modern dentitions. The archaeological sample from both studies is derived from The Schreiner Collection housed at the University of Oslo (Evensen and Øgaard 2007; Lindsten et al. 2002). Presumably both studies use the same collection because researchers examined similar criteria, including time period, place of origin, and accessibility. Additionally, R. Øgaard is a researcher for both projects. Consisting of remains from $14^{\text {th }}$ to $16^{\text {th }}$ century Oslo, Evensen and Øgaard (2007) scored each dentition with the Norwegian Need for Orthodontic Treatment Index (NOTI) and compared scores with those taken from a previous study of the dental casts of living children from the same area. Lindsten and colleagues (2002) compared the skeletal data with a previously collected data from from children of the Northern Norway Sami (1980s) as well as that from Oslo children (1960s and 1980s). In this study, they examined arch width and depth as a predictor for malocclusion (Lindsten et al. 2002). Once again, neither set of authors delved into the complexities of how Norwegian food has changed, only that it has generally become much easier to chew since the $14^{\text {th }}$ and $16^{\text {th }}$ centuries (Lindsten et al. 2002). Despite the lack of dietary information, the individuals studied have a known origin and there is a clear distinction between pre- and postIndustrial groups. As a result, these studies have an excellent basis from which to examine changes in occlusion.

Results from these investigations supported the authors' hypotheses: malocclusion had increased in Norway since the medieval period due to changes in diet affecting mastication (Evensen and Øgaard 2007; Lindsten et al. 2002). When comparing dentitions on the basis of NOTI, Evensen and Øgaard (2007) found that there had been a substantial increase in severity and 
frequency of malocclusions. Furthermore, the authors found no sex-based differences, which overall supports their hypothesis that malocclusion is a reaction to a change in diet and not an inherently biological trend. Evensen and Øgaard (2007:712) recorded malocclusion in 65\% of the modern sample, whereas only $36 \%$ of the medieval population showed signs of malocclusion, which is indicative of the secular trend in misalignment. In turn, Lindsten and colleagues (2002) found that the modern Oslo children were more at risk than any other group for developing malocclusion. In general, the skeletal population had much shallower arch depths, indicating that they experienced more masticatory stimulation from diet than modern children. This deeper arch depth places the modern children at risk for malocclusion. Furthermore, this would seem to agree with previous findings regarding "long-face" morphology associated with weak bite force: a deeper arch would facilitate this appearance to match with the posteriorly rotated mandible (Ingervall and Bitsanis 1987; Rando, Hillson, and Antoine 2014). These authors present strong evidence that when Norway experienced the Industrial Revolution, the corresponding softening of food resulted in a secular change in occlusion.

\section{Criteria for Skeletal Evaluation}

Analysis of the dietary effect on occlusion has interesting implications for modern studies of microevolution, but can also be useful for assessing archaeological remains of unknown subsistence. Based on the information reviewed in this paper, I propose the existence of orofacial characteristics to investigate archaeological assemblages or individuals. These characteristics could include metric measurements and qualitative analysis of bite alignment. While this would require additional testing in order to substantiate, there is reason to believe that one can infer information about the diet consumed during development based on characteristics such as these in combination with other dietary reconstruction methods (Sella-Tunis et al. 2018). Because it is not always abundantly clear how a group subsisted, this has use in substantiating claims regarding diet and food processing made on the basis of stable isotope analysis and archaeological interpretations. I believe this would be especially pertinent in re-evaluations of groups which have previously been classified as hunter-gatherer or agricultural. There are many subtle nuances to human subsistence, and it is abundantly clear that these two niches are not mutually exclusive or as straight-forward as has been previously imagined. Thus, looking at malocclusion and mandibular morphology can elucidate practices regarding food type and processing that have been suggested by other facets of archaeological study. Furthermore, in socially stratified societies, differences in food consistency and processing can often indicate relative socioeconomic status. Evaluation of these dental and orofacial characteristics may help determine an individual's position within the community, especially if the culture's food traditions are known. Though this is a promising area of future study, at present these criteria have not undergone any testing for palaeodietary reconstruction, and therefore are not directly associated with specific measurements or formulae.

Additionally, there are a few cautions in using these methods. One should always ensure that collaboration is a part of dietary study. By using all methods, resources, and researchers available, the analysis will be holistic and robust. Additionally, use of teeth as extra-masticatory tools can pose problems for analyzing these 
dental characteristics. With these caveats, it is possible to include this non-destructive study in the suite of methods used to determine paleodietary practices.

The following are orofacial characteristics observed on skeletal remains that could contribute to reconstructing diet in the past: depth of the COS, gonial angle, advanced wear, and malocclusion. These traits have proven to be the most plastic orofacial characteristics that change malocclusion as a result of masticatory forces and are best applied to a population or used in a larger reference sample. COS and gonial angle can be measured with simple metrics on dry bone, or on digital renderings of the mandible. Ideally, geometric morphometric analysis would be applied so as to control for body size differences, which may potentially be linked to sexual dimorphism. Advanced wear should be considered a qualitative analysis, using a scoring system such as the one outlined in Buikstra and Ubelaker (1994) to score cheek teeth. This needs to be controlled for age at death, and should only be loosely correlated with a rough, abrasive, or otherwise difficult to masticate diet versus soft and easy to chew diet. Malocclusion should be scored based on whichever clinical method best fits the region of study for modern comparatives. This can include Angle's classes of malocclusion, arch relationships, tooth crowding, tipping, rotation, and impaction (Varrella 1990). Applied correctly with the right collaborative dietary evidence, these craniodental features may be able to serve as evidence of dietary texture during development in archaeological populations.

\section{Future Research}

Research into the microevolution of the dentition is of great interest today. Evolution and development (evo-devo) studies seek to understand the functional integration of human jaws and teeth in order to explain why malocclusion occurs, and implement a healthcare plan in order to combat its development (Boughner 2017). Further work into what can be included in a child's diet and when this is most necessary should be conducted: does the food need to be tough, or abrasive? Does this correlate with how long a child is breastfed? Additionally, this research could be enriched with a treatment of how tooth size interacts with occlusion. Tooth size, morphology, and growth are genetically controlled, but there is little evidence for how that could be affected by the biomechanical forces that shape jaw development (Boughner and Dean 2004; Corruccini 1984).

Lastly, there is correlation with the epidemiological transition in occlusion with third molar exclusion (Boughner 2017; Sengupta et al. 1999). Examining this is out of the scope of this paper; however, the evolution of the third molar is of great interest to micro-evolution and evodevo studies of dentition (Boughner and Dean 2004; Marchiori et al. 2016). Is third molar exclusion through impaction or agenesis related to the forces that influence malocclusion? Or is it related to thresholding and the evolution of tooth size? Studying the interaction between environmental influences, genetic influences, epigenetics, and the evolution of resultant orofacial phenotypes gives rise to these questions and many more, as the field develops.

\section{Concluding Remarks}

There is a clear connection with masticatory strain generated by diet during development and the etiology of malocclusion. This is reflected in changes observed in archaeological populations, living humans, and experiments with animal models. Characteristics of the mandible and dentition can provide insight into the kinds of diet consumed during an individual's growth and 
development. This is important in understanding why modern post-Industrial malocclusion is drastically increased in both prevalence and severity from what is seen in pre-Industrial populations. In general, pre-Industrial societies practiced non-intensive processing of food, which led to a tough diet requiring heavy mastication. With the advent of intense processing, food became much easier to masticate, and so rates of malocclusion increased so drastically that it has been called an epidemiological transition (Corruccini 1984). This trend is seen in virtually every place where the dietary transition has occurred. Because this transition is largely due to technological adaptations, not every society has undergone this change in the same way, or at all (Corruccini 1984; Rando, Hillson, and Antoine 2014). Secular trends in malocclusion are fundamental in understanding the etiology of the aesthetic and health issues that stem from it today. By using an anthropological approach to dentofacial misalignment in the archaeological record and the present, we can evaluate how evolutionary pressures are currently acting on the modern human dentition.

\section{Acknowledgements}

I would like to thank the editors and anonymous reviewers for their much appreciated contributions. Without their hard work this would not have been possible.

\section{References Cited}

Baydaş, B., İbrahim Y., Nesrin A., İsmail C., and İlhan M. D. 2004. Investigation of the Changes in the Positions of Upper and Lower Incisors, Overjet, Overbite, and Irregularity Index in Subjects with Different Depths of Curve of Spee. The Angle Orthodontist 74 (3): 349-55. https://doi.org/10.1043/00033219(2004)074<0349:IOTCIT>2.0.CO;2.

Boughner, J.C. 2017. Implications of Vertebrate Craniodental Evo-Devo for Human Oral Health.. Journal of Experimental Zoology Part B: Molecular and Developmental Evolution 328 (4): 321-33. https://doi.org/10.1002/jez.b.22734.
Boughner, Julia C, and M. Christopher Dean. 2004. Does Space in the Jaw Influence the Timing of Molar Crown Initiation? A Model Using Baboons (Papio Anubis) and Great Apes (Pan Troglodytes, Pan Paniscus). Journal of Human Evolution 46 (3): 253-75. https://doi.org/10.1016/j.jhevol.2003.11.007.

Buikstra J.E. and Ubelaker D.H. 1994. Standards for Data Collection from Human Skeletal Remains. Fayetteville: Arkansas Archaeological Survey.

Ciochon, R. L., R. A. Nisbett, and R. S. Corruccini. 1997. Dietary Consistency and Craniofacial Development Related to Masticatory Function in Minipigs. Journal of Craniofacial Genetics and Developmental Biology 17 (2): 96-102.

Cleveland Museum of Natural History. 2018. Collections and Database. https://www.cmnh.org/physanthro/collection-database (accessed May 28, 2018).

Corruccini, R.S. 1984. An Epidemiologic Transition in Dental Occlusion in World Populations. American Journal of Orthodontics 86 (5): 419-26. https://doi.org/10.1016/S0002-9416(84)90035-6.

Eguchi, S., G.C. Townsend, L.C. Richards, T. Hughes, and K. Kasai. 2004. Genetic Contribution to Dental Arch Size Variation in Australian Twins. Archives of Oral Biology 49 (12): 1015-24. https://doi.org/10.1016/j.archoralbio.2004.07.006.

Evensen, J.P., and B. Øgaard. 2007. Are Malocclusions More Prevalent and Severe Now? A Comparative Study of Medieval Skulls from Norway. American Journal of Orthodontics and Dentofacial Orthopedics 131 (6): 710-16. https://doi.org/10.1016/j.ajodo.2005.08.037.

Feldens, Carlos Alberto, Angela Isabel dos Santos Dullius, Paulo Floriani Kramer, Annarosa Scapini, Adair Luiz Stefanello Busato, and Fabiana Vargas-Ferreira. 2015. Impact of Malocclusion and Dentofacial Anomalies on the Prevalence and Severity of Dental Caries among Adolescents. The Angle Orthodontist 85 (6): 1027-34. https://doi.org/10.2319/100914-722.1.

Fueki, K., E. Yoshida, K. O., and Y. Igarashi. 2013a. Association between Occlusal Curvature and Food Comminution and Mixing in Human Young Adults with Permanent Dentitions. Archives of Oral Biology 58 (4): 377-83. https://doi.org/10.1016/j.archoralbio.2012.06.006.

— 2013b. Association between Occlusal Curvature and Masticatory Movements with Different Test Foods in Human Young Adults with Permanent Dentitions. Archives of Oral Biology 58 (6): 674-80. https://doi.org/10.1016/j.archoralbio.2012.12.006.

Harris, E.F. 2008. Interpreting Heritability Estimates in the Orthodontic Literature. Seminars in Orthodontics, Genetics and Orthodontics, 14 (2): 125-34. https://doi.org/10.1053/j.sodo.2008.02.003.

Harris, E.F., and M.G. Johnson. 1991. Heritability of Craniometric and Occlusal Variables: A Longitudinal Sib Analysis. American Journal of Orthodontics and Dentofacial Orthopedics 99 (3): 258-268. 
Human Skull Side View - /signs_symbol/skull/Human _skull_side_view.png.html. Eye Diagram - /medical/ anatomy/eye/eye_diagram.png.html. Accessed August 13, 2018. https://www.wpclipart.com/signs_symbol/ skull/Human_skull_side_view.png.html.

Ingervall, B. and E. Bitsanis. 1987. A Pilot Study of the Effect of Masticatory Muscle Training on Facial Growth in Long-Face Children. European Journal of Orthodontics 9 (1): 15-23. https://doi.org/10.1093/ejo/9.1.15.

Kaifu, Y., Kazutaka K., G.C. Townsend, and L.C. Richards. 2003. Tooth Wear and the 'Design' of the Human Dentition: A Perspective from Evolutionary Medicine. American Journal of Physical Anthropology 122 (S37): 47-61. https://doi.org/10.1002/ajpa.10329.

Katz, David C., Mark N. Grote, and Timothy D. Weaver. 2017. Changes in Human Skull Morphology across the Agricultural Transition Are Consistent with Softer Diets in Preindustrial Farming Groups. Proceedings of the National Academy of Sciences 114 (34): 9050-55. https://doi.org/10.1073/pnas.1702586114.

King, L., E.F. Harris, and E.A. Tolley. 1993. Heritability of Cephalometric and Occlusal Variables as Assessed from Siblings with Overt Malocclusions. American Journal of Orthodontics and Dentofacial Orthopedics 104 (2): 121-31. https://doi.org/10.1016/S0889-5406(05)810017.

Komlos, John, and Brian A'Hearn. 2017. Hidden Negative Aspects of Industrialization at the Onset of Modern Economic Growth in the U.S. Structural Change and Economic Dynamics 41 (June): 43-52. https://doi.org/10.1016/j.strueco.2017.03.001.

Laird, M.F., N.E. Holton, J.E. Scott, R.G. Franciscus, S.D. Marshall, and T.E. Southard. 2016. Spatial Determinants of the Mandibular Curve of Spee in Modern and Archaic Homo. American Journal of Physical Anthropology 161 (2): 226-36. https://doi.org/10.1002/ajpa.23020.

Larsson E., B. Øgaard, R. Lindsten, N. Holmgren, M. Brattberg, and L. Brattberg. 2005. Craniofacial and Dentofacial Development in Pigs Fed Soft and Hard Diets. American Journal of Orthodontics and Dentofacial Orthopedics 128 (6): 731-39. https://doi.org/10.1016/j.ajodo.2004.09.025.

Ledogar, Justin A., Paul C. Dechow, Qian Wang, Poorva H. Gharpure, Adam D. Gordon, Karen L. Baab, Amanda L. Smith, et al. 2016. Human Feeding Biomechanics: Performance, Variation, and Functional Constraints. PeerJ 4 (July): e2242. https://doi.org/10.7717/peerj.2242.

Lieberman, D.E, G.E. Krovitz, F.W. Yates, M. Devlin, and M. St. Claire. 2004. Effects of Food Processing on Masticatory Strain and Craniofacial Growth in a Retrognathic Face. Journal of Human Evolution 46 (6): 655-77. https://doi.org/10.1016/j.jhevol.2004.03.005.
Limme, M. 2010. Diversification Alimentaire et Développement Dentaire : Importance Des Habitudes Alimentaires Des Jeunes Enfants Pour La Prévention de Dysmorphoses Orthodontiques. Archives de Pédiatrie, Diversification alimentaire de l'enfant, 17 (Supplement 5): S213-19. https://doi.org/10.1016/S0929693X(10)70930-1.

Lindsten, R., B. Ögaard, E. Larsson, and K. Bjerklin. 2002. Transverse Dental and Dental Arch Depth Dimensions in the Mixed Dentition in a Skeletal Sample from the 14th to the 19th Century and Norwegian Children and Norwegian Sami Children of Today. The Angle Orthodontist 72 (5): 439-48. https://doi.org/10.1043/00033219(2002)072<0439:TDADAD>2.0.CO;2.

Marchiori, Denver F., Garnet V. Packota, and Julia C. Boughner. 2016. Third-Molar Mineralization as a Function of Available Retromolar Space. Acta Odontologica Scandinavica 74 (7): 509-17. https://doi.org/10.1080/00016357.2016.1209240.

Marshall, S. D., Caspersen, M., Hardinger, R. R., Franciscus, R. G., Aquilino, S. A., and Southard, T. E. 2008. Development of the curve of Spee. American Journal of Orthodontics and Dentofacial Orthopedics, 134(3), 344-352. https://doi.org/10.1016/j.ajodo.2006.10.037

MouseDoctor. Jaw Position Affects Brain Blood Flow. Multiple Sclerosis Research. January 01, 1970. Accessed August 13, 2018. http://multiple-sclerosisresearch.blogspot.com/2014/07/jaw-position-affectsbrain-blood-flow.html.

Naini, Farhad B., James P. Moss, and Daljit S. Gill. 2006. The Enigma of Facial Beauty: Esthetics, Proportions, Deformity, and Controversy. American Journal of Orthodontics and Dentofacial Orthopedics 130 (3): 277-82. https://doi.org/10.1016/j.ajodo.2005.09.027.

Normando, D. J. Faber, J.F. Guerreiro, and C. Cardoso Abdo Quintão. 2011. Dental Occlusion in a Split Amazon Indigenous Population: Genetics Prevails over Environment. PLOS ONE 6 (12): e28387. https://doi.org/10.1371/journal.pone.0028387.

Pinhasi, R., V. Eshed, and N. von Cramon-Taubadel. 2015. Incongruity between Affinity Patterns Based on Mandibular and Lower Dental Dimensions Following the Transition to Agriculture in the Near East, Anatolia and Europe. PLOS ONE 10 (2): e0117301. https://doi.org/10.1371/journal.pone.0117301.

Rando, C., S. Hillson, and D. Antoine. 2014. Changes in Mandibular Dimensions during the Mediaeval to PostMediaeval Transition in London: A Possible Response to Decreased Masticatory Load. Archives of Oral Biology 59 (1): 73-81. https://doi.org/10.1016/j.archoralbio.2013.10.001. 
Rosenstiel, Stephen F., and Robert G. Rashid. 2002. Public Preferences for Anterior Tooth Variations: A WebBased Study. Journal of Esthetic and Restorative Dentistry 14 (2): 97-106. https://doi.org/10.1111/j.1708-8240.2002.tb00158.x.

Sarver, David M. 2001. The Importance of Incisor Positioning in the Esthetic Smile: The Smile Arc. American Journal of Orthodontics and Dentofacial Orthopedics 120 (2): 98-111. https://doi.org/10.1067/mod.2001.114301.

Scheuer, Louise., and Sue M Black.2004. The Juvenile Skeleton. London: Elsevier Academic Press

Sella-Tunis, Tanya, Ariel Pokhojaev, Rachel Sarig, Paul O’Higgins, and Hila May. 2018. Human Mandibular Shape Is Associated with Masticatory Muscle Force. Scientific Reports 8 (1). https://doi.org/10.1038/s41598018-24293-3.

Sengupta, A., Whittaker, D. K., Barber, G., Rogers, J., and Musgrave, J. H. 1999. The effects of dental wear on third molar eruption and on the curve of Spee in human archaeological dentitions. Archives of Oral Biology, 44(11), 925-934. https://doi.org/10.1016/S00039969(99)00095-3

Smith, Richard J., and Howard L. Bailit. 1977. Problems and methods in research on the genetics of dental occlusion. The Angle Orthodontist 47, no. 1: 65-77.

Tobias, Phillip V. 1980. The Natural History of the Helicoidal Occlusal Plane and Its Evolution in Early Homo. American Journal of Physical Anthropology 53 (2): 173-87. https://doi.org/10.1002/ajpa.1330530202.

Townsend, G., Toby H., Michelle L., Michelle B., and Alan B.. 2009. Genetic and Environmental Influences on Human Dental Variation: A Critical Evaluation of Studies Involving Twins. Archives of Oral Biology 54 (December): S45-51. https://doi.org/10.1016/j.archoralbio.2008.06.009.

Varrela, J. 1990. Occurrence of Malocclusion in Attritive Environment: A Study of a Skull Sample from Southwest Finland. European Journal of Oral Sciences 98 (3): 242-47. https://doi.org/10.1111/j.16000722.1990.tb00968.x.

von Cramon-Taubadel, Noreen von. 2011. Global Human Mandibular Variation Reflects Differences in Agricultural and Hunter-Gatherer Subsistence Strategies. Proceedings of the National Academy of Sciences 108 (49): 19546-51. https://doi.org/10.1073/pnas.1113050108.

Whatwhenhow RRS. The Permanent Maxillary Incisors (Dental Anatomy, Physiology and Occlusion) Part 2. Accessed August 13, 2018. http://what-whenhow.com/dental-anatomy-physiology-andocclusion/the-permanent-maxillary-incisors-dentalanatomy-physiology-and-occlusion-part-2/
Whatwhenhow RSS. The Permanent Mandibular Molars (Dental Anatomy, Physiology and Occlusion) Part 3. Accessed August 13, 2018. http://what-whenhow.com/dental-anatomy-physiology-andocclusion/the-permanent-mandibular-molars-dentalanatomy-physiology-and-occlusion-part-3/.

Witt, Matthew, and Carlos Flores-Mir. 2011. Laypeople's Preferences Regarding Frontal Dentofacial Esthetics: Tooth-Related Factors. The Journal of the American Dental Association. 